cytarabine plus mitoxantrone and postremission therapy by either autologous stem-cell transplantation or by prolonged maintenance for acute myeloid leukemia. J Clin Oncol 2006; 24: 2480-2489.

6 Yanada M, Borthakur G, Ravandi F, Bueso-Ramos C, Kantarjian H, Estey E. Kinetics of bone marrow blasts during induction and achievement of complete remission in acute myeloid leukemia. Haematologica 2008; 93 1263-1265.

7 Gajjar A, Ribeiro R, Hancock ML, Rivera GK, Mahmoud H, Sandlund JT et al. Persistence of circulating blasts after 1 week of multiagent chemotherapy confers a poor prognosis in childhood acute lymphoblastic leukemia. Blood 1995; 86: 1292-1295.

8 Gianfaldoni G, Mannelli F, Baccini M, Antonioli E, Leoni F, Bosi A. Clearance of leukaemic blasts from peripheral blood during standard induction treatment predicts the bone marrow response in acute myeloid leukaemia: a pilot study. Br J Haematol 2006; 134: 54-57.

9 Elliott MA, Litzow MR, Letendre LL, Wolf RC, Hanson CA, Tefferi A et al. Early peripheral blood blast clearance during induction chemotherapy for acute myeloid leukemia predicts superior relapse-free survival. Blood 2007; 110: $4172-4174$
10 Gianfaldoni G, Mannelli F, Bencini S, Leoni F, Baldini S, Bosi A. Peripheral blood blast clearance during induction therapy in acute myeloid leukemia. Blood 2008; 111: 1746-1747.

11 Lacombe F, Arnoulet C, Maynadie M, Lippert E, Luquet I, Pigneux A et al. Early clearance of peripheral blasts measured by flow cytometry during the first week of AML induction therapy as a new independent prognostic factor: a GOELAMS study. Leukemia 2009; 23: 350-357.

12 Arellano M, Pakkala S, Langston A, Tighiouart M, Pan $\mathrm{L}$, Chen $\mathrm{Z}$ et al. Early clearance of peripheral blood blasts predicts response to induction chemotherapy in acute myeloid leukemia. Cancer 2012; 118: 5278-5282.

13 Grimwade D, Hills RK, Moorman AV, Walker H, Chatters S, Goldstone AH et al. Refinement of cytogenetic classification in acute myeloid leukemia: determination of prognostic significance of rare recurring chromosomal abnormalities among 5876 younger adult patients treated in the United Kingdom Medical Research Council trials. Blood 2010; 116: 354-365.

14 Deeks JJ. Systematic reviews in health care: Systematic reviews of evaluations of diagnostic and screening tests. BMJ 2001; 323: 157-162.

15 Gatsonis C, Paliwal P. Meta-analysis of diagnostic and screening test accuracy evaluations: methodologic primer. AJR Am J Roentgenol 2006; 187: 271-281.

\title{
Global phosphoproteome analysis of human bone marrow reveals predictive phosphorylation markers for the treatment of acute myeloid leukemia with quizartinib
}

\section{Leukemia (2014) 28, 716-719; doi:10.1038/leu.2013.347}

Treatment with inhibitors of the receptor tyrosine kinase FLT3 are currently studied as promising therapies in acute myeloid leukemia (AML). However, only a subset of patients benefit from these treatments and the presence of activating mutations within FLT3 can predict response to a certain extent only. AC220 (quizartinib) is an example of a potent FLT3 inhibitor ${ }^{1}$ that was studied in a recent phase II open-label study in patients with relapsed/refractory AML. The complete remission rate (including CRp and CRi) in FLT3-ITD-positive patients was 54\% (50/92) and the corresponding partial remission rate (PR) was $17 \%(16 / 92)^{2}$ Thus, although the FLT3-ITD mutation status correlates with response, the error rate in stratification of patients into responders and non-responders is high, as still 29\% of the FLT3-ITD-positive patients failed to respond. Exclusion of FLT3-ITD-negative patients from AC220 treatment also seems critical, as the total response rate $(C R+P R)$ in FLT3-ITD-negative patients is substantially lower $(41 \%, 17 / 41)$. As AC220 is a tyrosine kinase inhibitor, we hypothesized that investigating phosphorylation-based signaling on a system-wide scale in AML cells allows for identification of markers enabling more accurate prediction of therapy response as compared to commonly used genetic markers. Hence, we applied quantitative mass spectrometry to decipher a multivariate phosphorylation site marker, which we refer to as phosphosignature, in patient-derived AML blasts that might be useful as predictive biomarkers for AC220 treatment.

We first collected bone marrow aspirates of 21 patients enrolled in the phase II clinical trial of AC220 monotherapy in AML (ACE, NCT00989261) with FLT3-ITD before treatment (Supplementary Table 1). We processed the aspirates according to a previously established sample preparation workflow (Figure 1 and Supplementary Methods). Twelve of the twenty-one samples were processed at the beginning of this study (training group) and were used to generate a training data-set for phosphosignature identification. Nine additional samples were processed toward the end of this study and were used for validating the phospho-signature (validation group). All patients with CR or PR were counted as responder in our study $(6 / 12$ in the training subgroup and $6 / 9$ in the validation subgroup).

To monitor quantitatively the phospho-proteomes of the patient-derived AML blasts, we used super-SILAC in combination with quantitative mass spectrometry (see Figure 1 and Supplementary Methods). Data analysis was finally performed by using the MaxQuant software ${ }^{3}$ and further bioinformatics tools as outlined below. In total, 13236 phospho-sites were identified in the training group. Of these, 7831 were confidently assigned to specific serine, threonine or tyrosine residues (class I sites).

We first investigated whether we can identify differentially regulated phospho-sites when comparing responder and nonresponder samples (Figure 2a). Only class I sites quantified in at least two thirds of the experiments were used (2119 sites with approximately $10.6 \%$ missing values on average). Indeed, application of the mean-rank test ${ }^{4}$ revealed three significantly different sites at a false-discovery rate of $10 \%$ (see Supplementary Table 2). The first regulated site (S160) is located on the endonuclease/ exonuclease/phosphatase family domain-containing protein 1 (EEPD1). The protein carrying the second phosphorylation site (S630) was B-cell lymphoma/leukemia 11A (BCL11A), which functions as a myeloid and B-cell proto-oncogene and may play a role in leukemogenesis and hematopoiesis. ${ }^{5}$ Furthermore, the expression of BCL11A is associated with a poor outcome of $A M L$ patients. ${ }^{6}$ The third phosphorylation site (S333) is located on Ranbinding protein 3 (RANBP3). RANBP3 mediates nuclear export of Smad $2 / 3$ and thereby inhibits TGF- $\beta$ signaling. ${ }^{7}$ Furthermore, the 


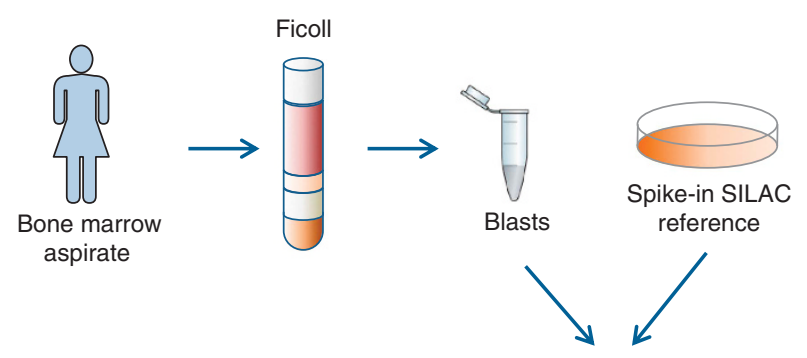

MaxQuant 7,831 Phospho-Sites
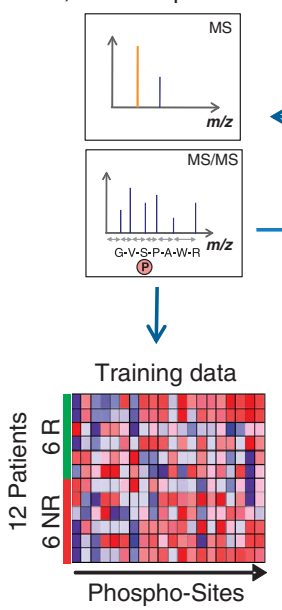

$(2,119)$

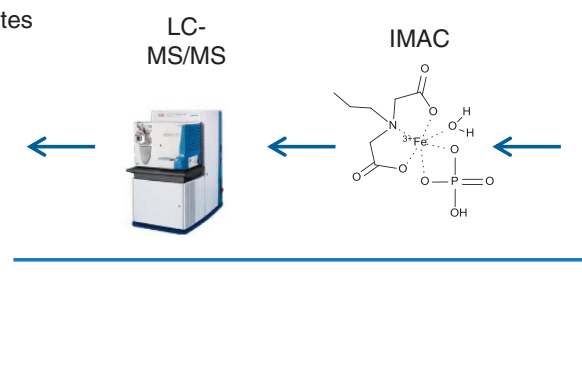

LC-

Trypsin digestion<smiles>C=C</smiles>

SCX chromatography

(12 fractions)

Figure 1. Workflow of processing bone marrow aspirates and global quantitative phosphoproteome analysis. The leukemia cells were isolated using density-gradient centrifugation and stored as vital cells for further processing at $-80^{\circ} \mathrm{C}$. Equal amounts of lysates from blasts and Super-SILAC-standard were mixed. Proteins were extracted and digested with trypsin. The resulting peptides were separated into 12 fractions by strong cation exchange (SCX) chromatography and the phosphopeptides were enriched using immobilized metal affinity chromatography (IMAC). High-resolution LC-MS/MS data were processed using the MaxQuant software. Data from 12 patients (six responders and six nonresponders) were used in the classification workflow for selection of five predictive phosphorylation sites (phospho-signature) and for training of a support vector machine. Classification accuracy was estimated with leave-one-out cross-validation. Finally, the signature was applied to nine independent validation samples.

Ras/ERK/RSK and the PI3K/AKT signaling pathways regulate the activity of RANBP3. ${ }^{8}$ Both the pathways are activated in FLT3-ITDpositive cells. ${ }^{9}$ To our knowledge, no function has been described for these phospho-sites in AML so far. Interestingly, other phosphorylation events that are downstream of FLT3-ITD, such as phosphorylation of Y694 in STAT5A, were not differentially regulated between the responder and the non-responder group (Supplementary Figure 1). Hence, it appears that only certain signaling pathways downstream of FLT3-ITD are differentially regulated between responders and non-responders and these pathways might contribute to resistance-mediating bypass signaling.

Next, we sought to identify a phospho-signature that allows prediction of responsiveness using a supervised machine learning approach. We therefore applied our previously described workflow for detecting phospho-signatures. ${ }^{10}$ A detailed description of the bioinformatics workflow is outlined in the Supplementary Methods.

The resulting final phospho-signature consisting of five phosphorylation sites strongly separates the classes of responder and non-responder samples (Figures $2 \mathrm{~b}$ and c, Supplementary Figure 2 and Supplementary Table 2). Three of the five phosphorylation sites (EEPD1-S160, BCL11A-S630, RANBP3-S333) were already identified as significantly regulated between responder and non-responder samples. The fourth phosphorylation site (S961) is located on the x-linked retinitis pigmentosa GTPase regulator (RP3). RP3 is predicted to be a guaninenucleotide releasing factor and has a role in ciliogenesis. ${ }^{11}$ Lamins A/C (LMN1), which harbored the fifth site (S458) from the phospho-signature, form the nuclear lamina and has an important role in cell cycle-dependent regulation of nuclear structure and gene transcription. ${ }^{12}$ All five sites were identified and localized with high confidence $\left(P>0.98\right.$, see $M^{2}$ spectra in Supplementary Figure 3).

The prediction performance of the phospho-signature was determined by leave-one-out cross-validation. In each iteration of cross-validation, the selection of phospho-site features and the training of a support vector machine is repeated on the training set reduced by the respective test sample. Notably, all samples except one sample (AML008) were correctly classified (Figure 2b), corresponding to a prediction accuracy of $92 \%$. Similarly the area under the receiver operating characteristic curve is $88 \%$. Although in case of AML008 no remission was observed, this patient also harbored a FLT3-TKD (D835) mutation at disease progression following 4 months of therapy, indicating FLT3 was inhibited as the mechanism for clinical response, albeit less than protocol defined PR.

We finally applied the identified phospho-signature to test its predictive power on nine additional validation samples (Figure 2d). These samples were processed independently of the training samples. Notably, seven out of the nine samples were predicted correctly, just one responder (AML031) and one nonresponder (AML033) were misclassified. AML033 was a borderline candidate. Notably, the patient had FLT3-ITD-positive cells that were sensitive and cleared by the drug treatment. However, the patient eventually progressed with a FLT3 wild-type clone. Even if taking this ambiguous call into account, the resulting sensitivity on the validation samples is $83 \%$ and the specificity is $67 \%$. 

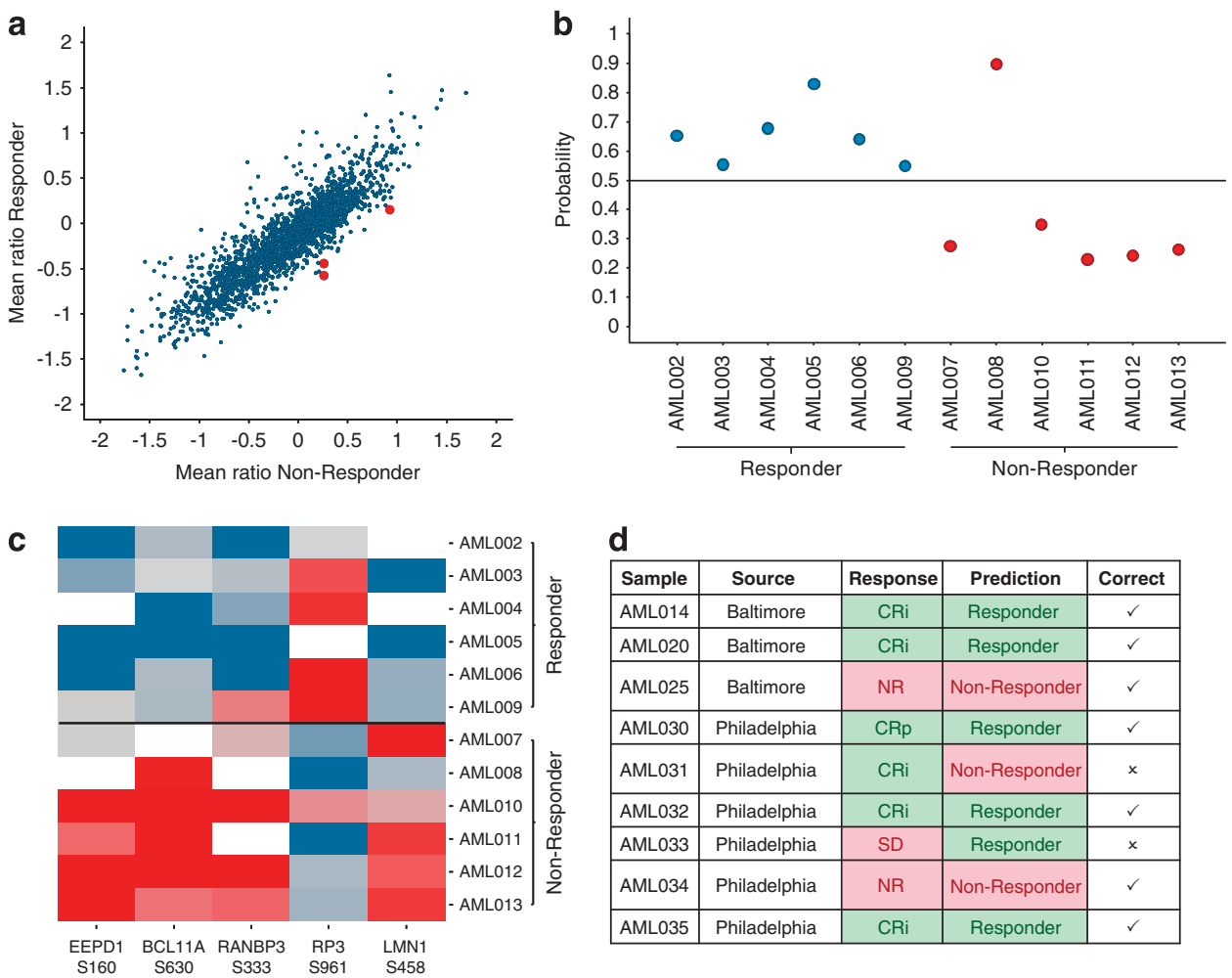

d
\begin{tabular}{|c|c|c|c|c|}
\hline Sample & Source & Response & Prediction & Correct \\
\hline AML014 & Baltimore & CRi & Responder & $\checkmark$ \\
\hline AML020 & Baltimore & CRi & Responder & $\checkmark$ \\
\hline AML025 & Baltimore & NR & Non-Responder & $\checkmark$ \\
\hline AML030 & Philadelphia & CRp & Responder & $\checkmark$ \\
\hline AML031 & Philadelphia & CRi & Non-Responder & $\times$ \\
\hline AML032 & Philadelphia & CRi & Responder & $\checkmark$ \\
\hline AML033 & Philadelphia & SD & Responder & $\times$ \\
\hline AML034 & Philadelphia & NR & Non-Responder & $\checkmark$ \\
\hline AML035 & Philadelphia & CRi & Responder & $\checkmark$ \\
\hline
\end{tabular}

Figure 2. Identification of predictive phospho-signature. (a) Scatter plot showing the mean log-ratios (AML sample vs spike-in SILAC reference) for the responder ( $y$ axis) and non-responder ( $x$ axis) samples. Each dot represents one phosphorylation site. The three significantly differential sites are marked red. (b) Cross-validation results represented by the probability for assignment to the responder class. Responders (left half) are predicted correctly if they are assigned a probability $>0.5$; non-responder (right half) are correct if they are assigned a probability $<0.5$. (c) Heat map of the final five selected phosphorylation sites. Rows are the 12 training sample, columns are the phospho-sites ordered by their importance ranks (left is the best). Red indicates up-, blue downregulation, gray no regulation. Missing values are colored white. (d) Validation results of nine independent patients. The true and predicted responses are compared.

The corresponding accuracy is $78 \%$ and therefore comparable to the accuracy determined in cross-validation. This is a promising result as the validation subgroup differed from the training subgroup both in terms of the source and in terms of the day of processing.

Differences in phosphorylation of a specific site may be caused by either a difference in phosphorylation site stoichiometry, a difference in expression of the corresponding protein, or by a combination of both. In order to distinguish between these three possibilities, we analyzed the proteome of six validation samples (Supplementary Figure 4). For two of the five signature proteins (EEPD1 and LMN1), we could quantify the predictive phosphorylation site and protein abundance in at least $2 / 3$ of the samples. LMN1 shows a very high correlation between phosphorylation and protein expression (Pearson correlation $r=0.92$, $P=0.03)$. The correlation for EEPD1 is smaller and not significantly different from $0(r=0.70, P=0.18)$ due to one outlier sample (Supplementary Figure 4A). Furthermore, although we enriched for phosphorylated peptides, we identified and quantified nonphosphorylated peptides of LMN1 in almost all training and validation samples. We could therefore correlate the phosphorylation of LMN1 with its expression in these samples (Supplementary Figure 4B) and again obtained a high correlation $(r=0.86$, $P=2.5 \times 10^{-6}$ ).

These results show the utility of a global and unbiased analysis to enable the identification of non-obvious but highly predictive markers that have no known association with the drug's main target. For clinical application of the biomarker signature, it would be sufficient to detect and quantify five phosphorylation sites. Notably, economic targeted detection methods, such as immunological methods or the mass spectrometry-based multiple reaction monitoring ${ }^{13}$ could be applied instead of global analysis strategies. Such targeted methods can reproducibly detect and quantify given peptides from relatively low sample amounts and can be routinely applied to large number of samples. We also showed that at least one of the phosphorylation markers, LMN1 (S458), strongly correlates with the expression of the corresponding protein. This creates the further option to measure LMN1 protein expression rather than performing targeted phosphorylation site analysis.

In summary, phosphoproteomic analyses of primary AML bone marrow by high-resolution quantitative mass spectrometry is feasible and offers the opportunity to discover posttranslational modifications as pre-therapeutic response parameters. A signature consisting of five phosphorylation sites predicted the response to treatment of AML patients with AC220.

\section{CONFLICT OF INTEREST}

CS, AT, HD and KG are employees and stock owners of Evotec. FSO and MK are employees of Evotec. AEP is consultant of Ambit Biosciences (unpaid) and Astellas (paid). ML is consultant of Astellas. The remaining authors declare no competing financial interest.

\section{ACKNOWLEDGEMENTS}

We are grateful to the colleagues at Evotec for excellent technical assistance We acknowledge Erich Enghofer and Michael Gorray (Bayer Vital, Leverkusen, Germany) for their help in initiating the study. This work is based on a project supported by the Federal German Ministry of Education and Research (0315011). 
C Schaab ${ }^{1,2}$, FS Oppermann ${ }^{1}$, M Klammer ${ }^{1}$, H Pfeifer $^{3}$, A Tebbe ${ }^{1}$, T Oellerich ${ }^{3,4,5}$, J Krauter ${ }^{6}, \mathrm{M}$ Levis $^{7}$, AE Perl $^{8}, \mathrm{H} \mathrm{Daub}^{1}$, B Steffen $^{3}$ $\mathrm{K} \mathrm{Godl}^{1}$ and $\mathrm{H}$ Serve $\mathrm{e}^{3,4,5}$

${ }^{1}$ Evotec (München) GmbH, Am Klopferspitz 19a, Martinsried, Germany;

${ }^{2}$ Max Planck Institute of Biochemistry, Am Klopferspitz 18, Martinsried, Germany;

${ }^{3}$ Department of Medicine, Hematology/Oncology, Goethe University, Theodor-Stern-Kai 7, Frankfurt, Germany; ${ }^{4}$ German Cancer Consortium (DKTK), Heidelberg, Germany; ${ }^{5}$ German Cancer Research Center (DKFZ), Heidelberg, Germany; ${ }^{6}$ Department of Medicine, Hematology/Oncology, Medizinische Hochschule Hannover, Hannover, Germany; ${ }^{7}$ Sidney Kimmel Comprehensive Cancer Center, Johns Hopkins University, Baltimore, MD, USA and

${ }^{8}$ Hematologic Malignancies Program, Abramson Cancer Center, University of Pennsylvania, Philadelphia, PA, USA E-mail: christoph.schaab@evotec.com or serve@em.uni-frankfurt.de

\section{REFERENCES}

1 Zarrinkar PP, Gunawardane RN, Cramer MD, Gardner MF, Brigham D, Belli B et al. AC220 is a uniquely potent and selective inhibitor of FLT3 for the treatment of acute myeloid leukemia (AML). Blood 2009; 114: 2984-2992.

2 Cortes JE, Perl AE, Dombret $\mathrm{H}$, Kayser S, Steffen B, Rousselot $\mathrm{P}$ et al. Final results of a phase 2 open-label, monotherapy efficacy and safety study of Quizartinib (AC220) in patients $\geq 60$ years of age with FLT3 ITD positive or negative relapsed/refractory. AML Blood (ASH Ann Meeting Abstr) 2012; 120: Abstract 48.

3 Cox J, Mann M. MaxQuant enables high peptide identification rates, individualized p.p.b.-range mass accuracies and proteome-wide protein quantification. Nat Biotechnol 2008; 26: 1367-1372.
4 Klammer M, Dybowksi JN, Hoffmann D, Schaab C. Identification of significant features by a global mean rank test. BMC Bioinfo 2013 (Submitted).

5 Saiki Y, Yamazaki Y, Yoshida M, Katoh O, Nakamura T. Human EVI9, a homologue of the mouse myeloid leukemia gene, is expressed in the hematopoietic progenitors and downregulated during myeloid differentiation of HL60 cells. Genomics 2000; 70: 387-391.

6 Yin B, Delwel R, Valk PJ, Wallace MR, Loh ML, Shannon KM et al. A retroviral mutagenesis screen reveals strong cooperation between $\mathrm{Bcl11a}$ overexpression and loss of the Nf1 tumor suppressor gene. Blood 2009; 113: 1075-1085.

7 Dai F, Lin X, Chang C, Feng XH. Nuclear export of Smad2 and Smad3 by RanBP3 facilitates termination of TGF-beta signaling. Dev Cell 2009; 16: 345-357.

8 Yoon SO, Shin S, Liu Y, Ballif BA, Woo MS, Gygi SP et al. Ran-binding protein 3 phosphorylation links the Ras and PI3-kinase pathways to nucleocytoplasmic transport. Mol Cell 2008; 29: 362-375.

9 Choudhary C, Olsen JV, Brandts C, Cox J, Reddy PN, Bohmer FD et al. Mislocalized activation of oncogenic RTKs switches downstream signaling outcomes. Mol Cell 2009; 36: 326-339.

10 Klammer M, Kaminski M, Zedler A, Oppermann F, Blencke S, Marx S et al. Phosphosignature predicts dasatinib response in non-small cell lung cancer. Mol Cell proteomics 2012; 11: 651-668.

11 Gakovic M, Shu X, Kasioulis I, Carpanini S, Moraga I, Wright AF. The role of RPGR in cilia formation and actin stability. Hum mol genet 2011; 20: 4840-4850.

12 Meier R, Muller PR, Hirt A, Leibundgut K, Ridolfi-Luthy A, Wagner HP. Differential phosphorylation of lamin B2 in normal and leukemic cells. Leuk Res 1997; 21: 841-847.

13 Kitteringham NR, Jenkins RE, Lane CS, Elliott VL, Park BK. Multiple reaction monitoring for quantitative biomarker analysis in proteomics and metabolomics. J Chromatogr B Analyt Technol Biomed Life Sci 2009; 877: 1229-1239.

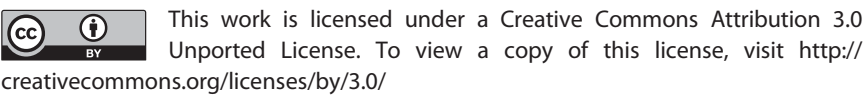
creativecommons.org/licenses/by/3.0/

\section{High prevalence of oncogenic $M Y D 88$ and $C D 79 B$ mutations in primary testicular diffuse large B-cell lymphoma}

Leukemia (2014) 28, 719-720; doi:10.1038/leu.2013.348

Diffuse large B-cell lymphoma (DLBCL) is a heterogeneous class of lymphomas, comprising of molecularly distinct subtypes that differ in gene-expression profile (GEP), genetic aberrations, clinical presentation and disease outcome., ${ }^{1,2}$ Within this lymphoma class, primary testicular lymphoma (PTL) is a distinctive entity characterized by unique clinical and molecular features, including its exclusive manifestation in the immune-privileged microenvironment of the testis and frequent dissemination to the contralateral testis and the central nervous system (CNS). ${ }^{3,4}$ Although the incidence of PTL has significantly increased over the last decades, there is at present no consensus on a standard therapeutic regimen., ${ }^{3,4}$ A current GEP-based molecular classification of DLBCL distinguishes two main subtypes: activated B-cell-like $(A B C)$ lymphoma and germinal-center B-celllike lymphoma. ${ }^{1}$ PTLs belong to the $A B C-D L B C L$ subtype that is characterized by constitutively active nuclear factor (NF) $-\kappa B$ signaling. ${ }^{1,2} \mathrm{NF}-\kappa \mathrm{B}$ pathway activation in DLBCL may result from oncogenic CARD11 mutations and/or of CD79 mutations causing chronic active B-cell receptor (BCR) signaling. ${ }^{5-7}$ In addition, somatically acquired mutations in MYD88, an adaptor protein that mediates toll-like receptor (TLR) and interleukin-1 receptor

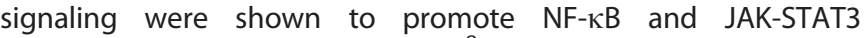
signaling in this lymphoma type. ${ }^{8}$ Intriguingly, recent studies indicate that the prevalence of oncogenic MYD88 mutations varies greatly among the $A B C-D L B C L$ presenting at different anatomical sites: whereas MYD88 mutations show a high prevalence in primary-CNS-lymphomas (PCNSLs) as well as in lymphomas arising at some other extra-nodal sites, they are relatively uncommon in primary nodal and gastro-intestinal DLBCL. ${ }^{9-11}$ In a survey of genomic alterations in a large panel of $D L B C L$, we recently found an activating MYD88 mutation in 10 out of 14 PTLs studied, ${ }^{11}$ suggesting a high mutation prevalence. Here we extended these series to obtain robust evidence for a role of deregulated MYD88 signaling in PTLs.

The study material comprised a panel of 37 PTL diagnosed as DLBCLs according the World Health Organization classification, 14 of which have been reported previously. ${ }^{11}$ All tumors were extensively immuno-phenotyped, including antibodies against CD20, CD10, MUM1, BCL-2 and BCL-6, and tested for Epstein-Barr virus (EBV) expression by EBV-encoded RNA in-situ hybridization, and tested for translocations of $B C L-2, B C L-6$ and $c-M Y C$ by fluorescence in-situ hybridization (Supplementary Table 1). ${ }^{11}$ To detect somatic mutations in MYD88 and CD79B, a panel of allele-specific PCRs covering all major mutation (hot) spots $^{8}$ was employed. As recently reported, this strategy permits efficient and sensitive detection of mutations using DNA extracted from the 